\title{
Long noncoding RNA TUG1 is downregulated in non-small cell lung cancer and can regulate CELF1 on binding to PRC2
}

Pei-Chin Lin ${ }^{1,8,9}$, Hsien-Da Huang ${ }^{3}$, Chun-Chi Chang ${ }^{1,10}$, Ya-Sian Chang ${ }^{2}$, Ju-Chen Yen², Chien-Chih Lee ${ }^{2}$, Wen-Hsin Chang ${ }^{6}$, Ta-Chih Liu ${ }^{1,6,7^{*}}$ and Jan-Gowth Chang ${ }^{2,4,5^{*}}$

\begin{abstract}
Background: Long noncoding RNAs (IncRNAs) play crucial roles in tumorigenesis, and IncRNA taurine-upregulated gene 1 (TUG1) has been proven to be associated with several human cancers. However, the mechanisms of TUG1involved regulation remain largely unknown.

Methods: We examined the expressions of TUG1 in a cohort of 89 patients with non-small cell lung cancer (NSCLC) to determine the association between TUG1 expression and clinical parameters. We used circular chromosome conformation capture (4C) coupled with next-generation sequencing to explore the genome regions that interact with TUG1 and the TUG1-mediated regulation.

Results: TUG1 was significantly downregulated, and the TUG1 downregulation correlated with sex $(p=0.006)$, smoking status $(p=0.016)$, and tumor differentiation grade $(p=0.001)$. Knockdown of TUG1 significantly promoted the proliferation of NSCLC cells. According to the bioinformatic analysis result of TUG1 4C sequencing data, 83 candidate genes and their interaction regions were identified. Among these candidate genes, CUGBP and Elav-like family member 1 (CELF1) are potential targets of TUG1 in-trans regulation. To confirm the interaction between TUG1 and CELF1, relative expressions of CELF1 were examined in TUG1 knockdown H520 cells; results showed that CELF1 was significantly upregulated in TUG1 knockdown H520 cells. RNA immunoprecipitation was then performed to examine whether TUG1 RNA was bound to PRC2, a TUG1-involved regulation mechanism reported in previous studies. The results demonstrated that TUG1 RNA was bound to enhancer of zeste protein 2/embryonic ectoderm development (EZH2/EED), which is essential for PRC2. Finally, our designed ChIP assay revealed that the EZH2/EED was bound to the promotor region of CELF1 within 992 bp upstream of the transcript start site.

Conclusion: TUG1 is downregulated in NSCLC. Using TUG1 4C sequencing and bioinformatic analysis, we found CELF1 to be a potential target of TUG1 RNA in in-trans regulation. Moreover, subsequent experiments showed that TUG1 RNA could bind to PRC2 in the promotor region of CELF1 and negatively regulate CELF1 expressions in H520 cells. Our results may facilitate developing new treatment modalities targeting TUG1/PRC2/CELF1 interactions in patients with NSCLC.
\end{abstract}

Keywords: Long noncoding RNA (InCRNA), Taurine-upregulated gene 1 (TUG1), Non-small cell lung cancer (NSCLC), CUGBP and Elav-like family member 1 (CELF1), Circular chromosome conformation capture (4C)

\footnotetext{
* Correspondence: d730093@kmu.edu.tw; d6781@mail.cmuh.org.tw

${ }^{1}$ Graduate Institute of Clinical Medicine, Kaohsiung Medical University, No.

100, Shih-Chuan 1st Road, Kaohsiung, Taiwan

²Epigenome Research Center, China Medical University Hospital, No. 2,

Yuh-Der Road, Taichung, Taiwan

Full list of author information is available at the end of the article
} 


\section{Background}

As a leading cause of cancer-related mortality worldwide, lung cancer has been investigated in numerous molecular genetic studies aimed at developing new treatment strategies [1]. Lung cancer is classified into two types according to biological characteristics: non-small cell lung cancer (NSCLC) (accounting for approximately $85 \%$ of cases), and small cell lung cancer (SCLC) [2]. The overall prognosis for lung cancer is poor; in 2004, the overall 5-year survival rate at all stages was $16.8 \%$ [1]. Small molecule tyrosine kinase inhibitors targeting receptor tyrosine kinases (RTKs), such as epidermal growth factor receptor (EGFR) and anaplastic lymphoma kinase (ALK), play crucial roles in NSCLC treatment. Nonetheless, prognosis and outcomes for patients with certain genetic features (e.g., EGFR mutations, K-Ras mutations, and EML4-ALK rearrangement) remain poor [3].

Long noncoding RNAs (lncRNAs), defined as ncRNAs with transcripts longer than 200 nucleotides, have a critical role in the development process, cellular homeostasis, genomic imprinting, and pluripotency of embryonic stem cells [4-6]. The importance of lncRNA regulation is emphasized by their roles in the etiology human diseases [7-9]. Several lncRNAs are involved in the carcinogenesis, disease progression, or metastasis of human cancers (e.g., MALAT1 in hepatocellular carcinoma, colorectal carcinoma, bladder cancer, and lung cancer; HOTAIR in breast cancer, hepatocellular carcinoma, pancreatic cancer, gastric cancer, laryngeal cancer, and nasopharyngeal cancer; H19 in cervical, gastric, bladder, breast, esophageal, and lung cancer; PCGEM1 in prostate cancer) [10].

The lncRNA taurine-upregulated gene 1 (TUG1) is a nonprotein-coding gene located on chromosome 22q12.2 that transcribes to a 6.7-kilobase-long, spliced, and polyadenylated RNA. Upregulated by taurine in developing retinal cells, TUG1 is essential for normal photoreceptor development. Knockdown of TUG1 leads to malformed outer segments of photoreceptors in newborn murine retinas [11]. In human cancers, TUG1 has been reported to be associated with urothelial carcinoma of the bladder, osteosarcoma, esophageal squamous cell carcinoma, and NSCLC [12-15]. Only a few studies have proposed the mechanisms of TUG1 regulation $[15,16]$. In the present study, we examined TUG1 expression in NSCLC patients to determine the association between TUG1 expressions and clinical parameters. LncRNAs regulate protein-coding gene expression through chromatin remodeling, transcriptional modulation, and nuclear architecture/subnuclear localization [17]. Chromosome conformation capture (3C) techniques are methods for detecting the coassociation between chromatins through the fixation of living cells, which preserves the genomic architecture in its native state before fragmentation by restriction enzyme digestion, and the ligation of chromatin fragments that are in physical proximity in the nuclear space [18]. Circular chromosome conformation capture (4C), which involves the circularization of chimeric DNA fragments and the amplification of DNA sequences with primers within the bait but proximal to the target sequence during ligation, can be used to screen for interactions without perception of the existence of two different complexes [19]. To further investigate TUG1 regulation, we used the $4 \mathrm{C}$ method to analyze genome-wide interactions with the TUG1 gene and found a novel target of TUG1 regulation.

\section{Methods \\ Patient samples}

Tumor and nontumor tissue samples were obtained from 89 patients with NSCLC. All participants provided written informed consent. The study was approved by the Institutional Review Board of Kaohsiung Medical University Hospital (KMUH-IRB-980524). The baseline characteristics of the patients with NSCLC (age, sex, smoking status, Eastern Cooperative Oncology Group performance status (ECOG PS), histology, differentiation grade, and TNM stage) were collected from chart records.

\section{RNA extraction and qRT-PCR}

Total RNA extraction, complementary DNA (cDNA) generation, and polymerase chain reaction (PCR) were performed according to manufacturer protocals. The detailed procedures and primer sequences are listed in Additional file 1. Glyceraldehyde-3-phosphate dehydrogenase (GAPDH) was used as an internal control.

\section{Cell culture and shRNA transfection}

H520, H1299, and REH cells were cultured in RPMI medium (GIBCO BRL, Gaithersburg, MD, USA) supplemented with $10 \%$ fetal bovine serum (GIBCO BRL) at $37{ }^{\circ} \mathrm{C}$ in a $5 \% \mathrm{CO}_{2}$ atmosphere. TUG1 shRNA, scramble RNA, and mock were obtained from the National Research Program for Biopharmaceuticals and were transfected into H520 and H1299 cells (400 ng TUG1 shRNA added to $600 \mu \mathrm{L}$ of cells, $2 \times 10^{5}$ cells $/ \mathrm{mL}$ ) by using Lipofectamine 2000 (Life Technologies). Transfection efficiency was determined through quantitative reverse transcription polymerase chain reaction (qRT-PCR).

\section{Cell proliferation assay}

Cell proliferation assay was used to examine whether TUG1 knockdown affects the viability of NSCLC cells. In brief, transfected $\mathrm{H} 520$ and $\mathrm{H} 1299$ cells were plated in 96-well plates $\left(2 \times 10^{5}\right.$ cells/mL, $100 \mu \mathrm{L} /$ well $)$. After $48 \mathrm{~h}$, cell proliferation and viability were examined using the MTT assay. All experiments were performed in triplicate. 


\section{Circular chromosome conformation capture}

The $4 \mathrm{C}$ experiment included the following basic procedures: formaldehyde cross-linking, and digestion and ligation of the known bait chromatin (i.e., TUG1 in this study) and the unknown sequences. Circular chimeric chromatin was then decross-linked, and the unknown sequences were amplified with inverse PCR by using the bait-specific primers. We followed the 4C method described by Stadhouders et al. [18], which involved secondary digestion and ligation between decross-linked and inverse PCR amplification. Secondary digestion is advantageous because it decreases the size of the DNA circles, enabling efficient PCR amplification of fragments. Six-base-recognizing (six-cutter) enzymes, which perform well on cross-linked chromatin, are generally recommended for primary digestion; any four-cutter enzyme that is insensitive to mammalian DNA methylation and has high religation efficiency can be used for secondary digestion. In addition, the final combination of primary and secondary restriction enzymes generates a suitable bait fragment for designing bait-specific primers for inverse PCR, depending on their compatibility [18]. Based on our bait gene sequence (TUG1), a sixcutter enzyme (HindIII) was used as the primary digestive enzyme, and a four-cutter enzyme (CviQI) that can generate a bait sequence suitable for further primer design in HindIII-digested TUG1 fragments was used as the secondary digestive enzyme. In brief, REH cells were cross-linked with $1 \%$ formaldehyde for $10 \mathrm{~min}$ at room temperature to preserve the three-dimensional nuclear architecture. HindIII was used to digest cross-linked chromatin (primary digestion). The digested chromatin was then ligated using the T4 DNA ligase. The digested and ligated chromatin was then decross-linked and submitted to the second restriction digestion by using CviQI to reduce the size of the fragments. Inverse PCR reactions were performed using TUG1-specific primers harboring Illumina adapter sequences to amplify the genomic DNA fragments ligated to TUG1 (first PCR: forward 5'-gtctccgatagtgcacacagc-3', reverse 5'-gaccatctccttcaggacca-3'; nested PCR: forward 5'-cattcagccaatcacaaagct-3', reverse 5'-cagatttatgacatagttccttccaa-3').

\section{Next-generation sequencing}

The PCR products were purified using the Qiagen MiniElute kit. After purification, the amplicon was prepared for sequencing by using a Truseq DNA library preparation kit (Illumina). According to the TruSeq DNA Sample Preparation protocol, 100-ng purified amplicon pools were repaired to generate blunt-ended, 5'-phosphorylated DNA, and an A-tailing reaction compatible with the adapter ligation strategy was performed. The ligation product was purified by sample purification beads. To enrich the library, an enhanced PCR mix was used to perform PCR amplification. The size distribution of the library was verified using the High-Sensitivity DNA Kit (Agilent), and the concentration of the library was quantified using the GeneRead Library Quant Kit (Qiagen). The library was diluted and sequenced with 500 pairedend cycles on the Illumina MiSeq by following the standard protocol.

\section{Bioinformatic analysis of TUG1 4C-sequencing data Removing known fragments from sequencing reads}

Removal of known fragments from sequencing reads involved three steps. First, forward and reverse sequencing reads were merged into one sequence when the length of the overlapping region between the forward and reverse sequencing reads was more than $20 \mathrm{nt}$. Second, BLAST, a widely used bioinformatic software of sequence searching, was used to identify the location of the known fragments and primers [20]. The known fragments were located in the regions between the primers and the cutting site of the enzymes. The alignment similarity for BLAST was set at $95 \%$. Finally, results from Blast showed the location of known fragments and primers. These primers and known fragments of sequencing reads were removed from the sequencing reads. The remaining region of the sequencing reads was labeled "unknown fragments."

\section{Identifying potential TUG1 interaction regions}

The Bowtie2 software is an efficient tool for aligning sequencing reads against reference sequences [21]. Bowtie2 was used to align unknown fragments against human genome sequences (Grch38.p2 was used in the present study). Subsequently, fourSig, a software suite for analyzing and visualizing $4 \mathrm{C}$-seq data, was used to identify the potential TUG1 interactive regions [22]. The command "bamToReTab.pl -H 210500300 bowtie2_output hind3_site.txt nla3_site.txt NONE > foursig_output" was used to obtain the primary results from fourSig. The primary results were then used to scan for potential interaction regions. The window size was set at 50 (i.e., 50 fragments in the window). After scanning for the interaction regions, fourSig provided three categories of regions, which were defined as potential TUG1 interaction regions. The regions and their associated genes were listed and then annotated according to the genomic location of known human genes. These TUG1 interactive regions, having over 100 reads of coverage, were used as TUG1 interaction region candidates.

\section{RNA immunoprecipitation (RIP)}

RNA immunoprecipitation (RIP) assays were performed using ChIP-IT (Active Motif, Carlsbad, CA, USA), according to the Active Motif protocol. Anti-EED (Aviva Systems Biology, San Diego, CA, USA), anti-EZH2 (Cell 
Signaling Technology, Danvers, MA, USA) antibodies, and IgG were used. The detailed procedures are listed in Additional file 2.

\section{DNA ChIP}

DNA ChIP assays were performed using ChIP-IT (Active Motif, Carlsbad, CA, USA), according to manufacturer instructions. Anti-EED (Aviva Systems Biology, San Diego, CA, USA), anti-EZH2 (Cell Signaling Technology, Danvers, MA, USA) antibodies, and IgG were used. The detailed procedures are listed in Additional file 2. Six sets of primers were designed to amplify the CELF1 promotor region. The primer sequences are listed in Additional file 3: Table S1.

\section{Statistical analysis}

The differences in RNA expression between tumor and nontumor tissues from NSCLC patients were analyzed using a paired $t$ test. The association between relative TUG1 RNA expression levels and clinical parameters (age, sex, smoking status, ECOG PS, histology, differentiation grade, and TNM stage) was analyzed using a $t$ test and ANOVA. TUG1 shRNA and scramble shRNA transfected cells in the MTT assay were compared and analyzed through an independent samples $t$ test. Statistical analyses were performed using SAS version 9.3; $p<0.05$ was considered statistically significant.

\section{Results}

\section{TUG1 is downregulated in NSCLC tissues and affects NSCLC cell prolifiration}

We determined the levels of TUG1 expression in 89 pairs of NSCLC tumor and nontumor lung tissues by using qRT-PCR. TUG1 was significantly downregulated in the cancerous tissues compared with the normal counterparts $(p<0.01)$ (Fig. 1a). Over $50 \%$ reduction in TUG1 expression was observed in $60 \%(35 / 58)$ of the TUG1-downregulated NSCLC tissues (Fig. 1b). Moreover, TUG1 downregulation correlated significantly with sex $(p=0.006)$, smoking status $(p=0.016)$, and tumor differentiation grade $(p=0.001)$ (Fig. 1c-e). Correlations with the other parameters (i.e., histology, age $(<65$ and $\geq 65$ years), and TNM stage) were nonsignificant (Additional file 4: Table S2). No differences were observed in the survival rates between patients with TUG1

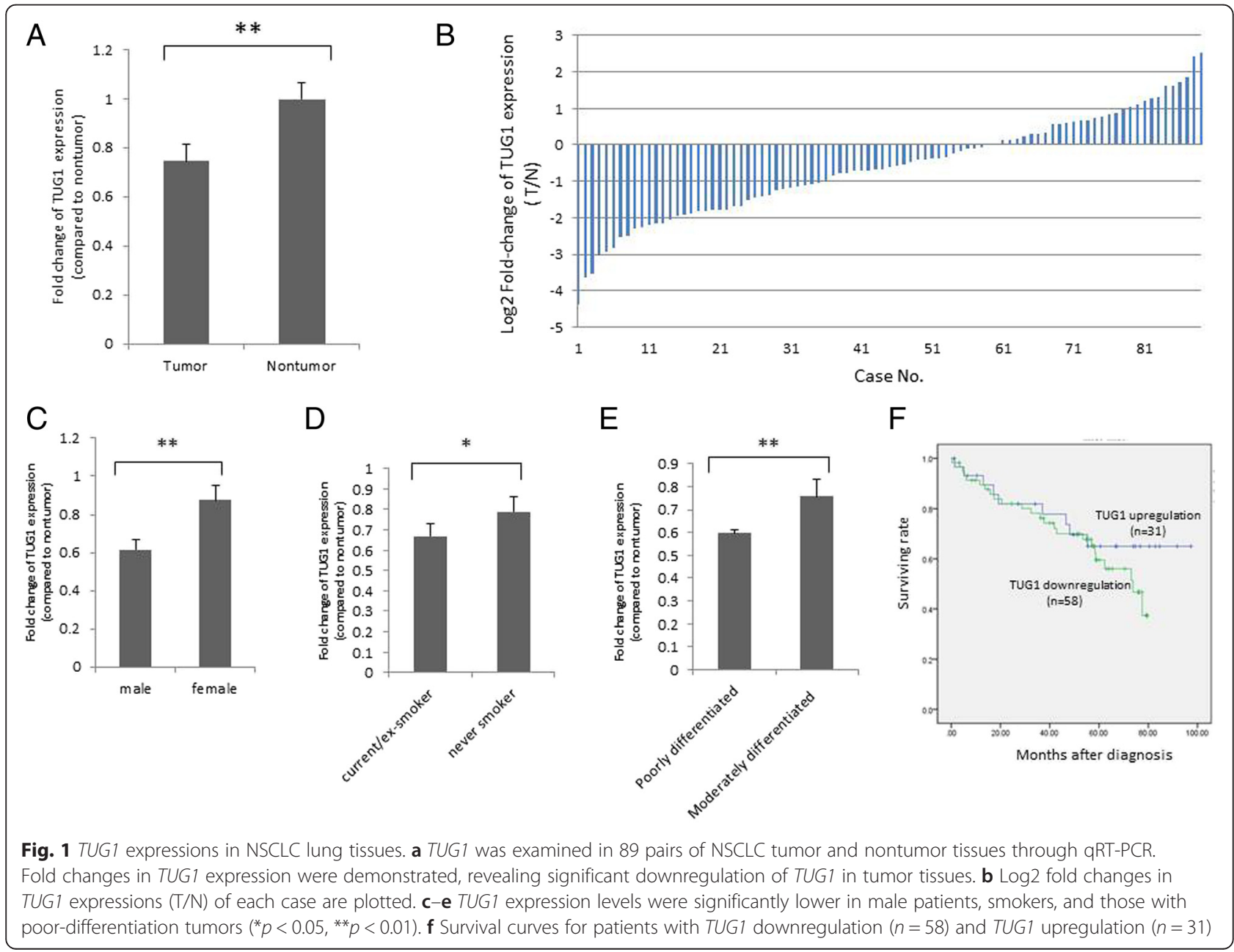


downregulation and those with TUG1 upregulation until 60 months after diagnosis, indicating that survival is lower among TUG1-downregulated patients compared with TUG1-upregulated patients (Fig 1f). To explore the effects of TUG1 regulation on cell proliferation, TUG1 knockdown was performed using shRNAs transfection in H520 and H1299 cells. The knockdown efficiencies were validated through qRT-PCR (Fig. 2a and b). The MTT assay showed that the TUG1 knockdown increased the cell proliferation in the H520 and H1299 cells (Fig. 2c and d).

CELF1 is a potential target of TUG1 interaction and could be negatively regulated by TUG1 RNA

LncRNAs have been shown to regulate their target genes by physically connecting their genomic locus with the genomic regions of the target genes [23]. To further investigate TUG1-involved regulation, we applied 4C coupled with next-generation sequencing procedures to identify the TUG1 interaction regions. The $4 \mathrm{C}$ experiment is a $3 \mathrm{C}$ based technique. 3C-based techniques have been applied in investigating lncRNA regulation mechanisms. Using highthroughput chromosome conformation capture, Wang et al. reported the necessity of induced proximity between the genomic region of HOTTIP and WDR5 in HOTTIP RNA regulation of its target genes [24]. Agarose gel electrophoresis of formaldehyde cross-linked, restriction-enzymedigested chromatin DNA, and PCR products amplified using TUG1-specific primers are illustrated in Additional file 5: Figure S1. The TUG1 4C-sequencing data had 6,302,180 paired-end sequencing reads. The data were deposited in NCBI SRA, project number: PRJNA293783. Figure 3 shows the analysis flowchart of the TUG1 data. According to the analysis results, 83 candidate genes were identified. These genes and their interaction regions are listed in Additional file 6: Tables S3 and S4. The TUG1 interaction map within these candidate genes is shown in
A

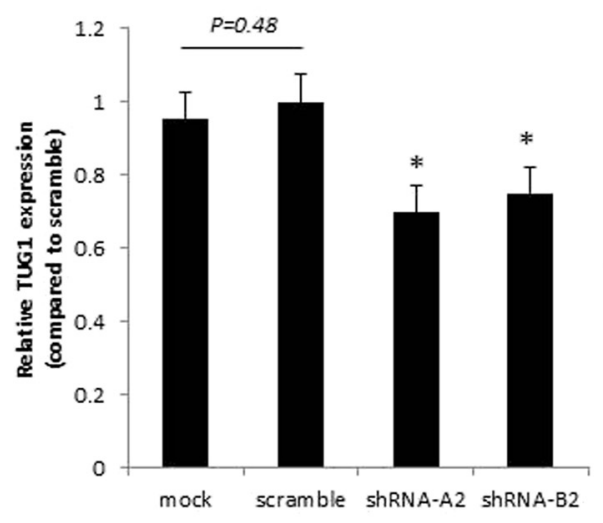

C

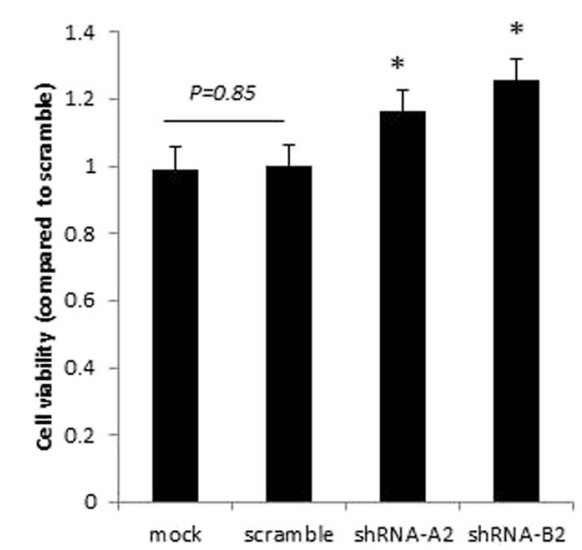

B

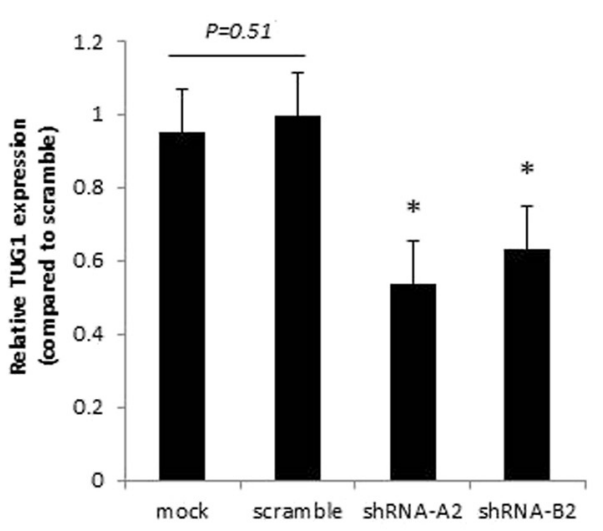

D

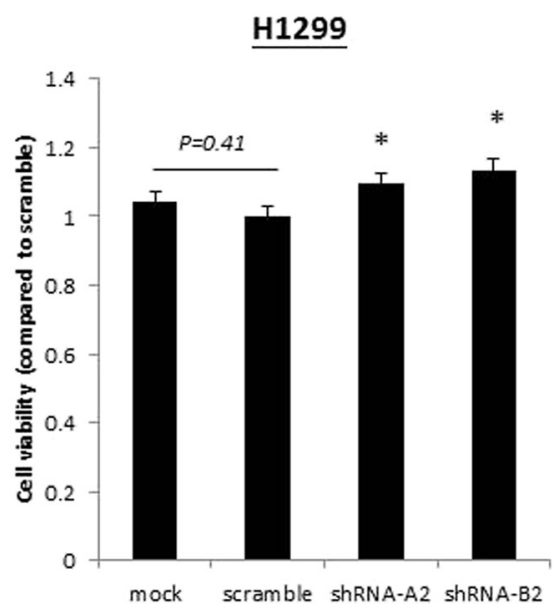

Fig. 2 Knockdown of TUG1 increases cell proliferation in NSCLC cells. NSCLC cells were transfected with shRNA A2 and B2 for the TUG1 knockdown, and scramble RNA was used as the control. The efficiencies of the TUG1 knockdown were assessed through real-time qRT-PCR in three independent experiments. $\mathbf{a}$ and (b) shRNA A2 and B2 demonstrated effective knockdown efficacy in H520 and H1299 cells. c and (d) Cell viabilities were examined in shRNA A2 and B2 knockdown H520 and H1299 cells. Significantly increased cell viabilities were noted compared with the scramble RNA in the three independent experiments $\left({ }^{*} p<0.05\right)$ 


\section{Analysis flow}

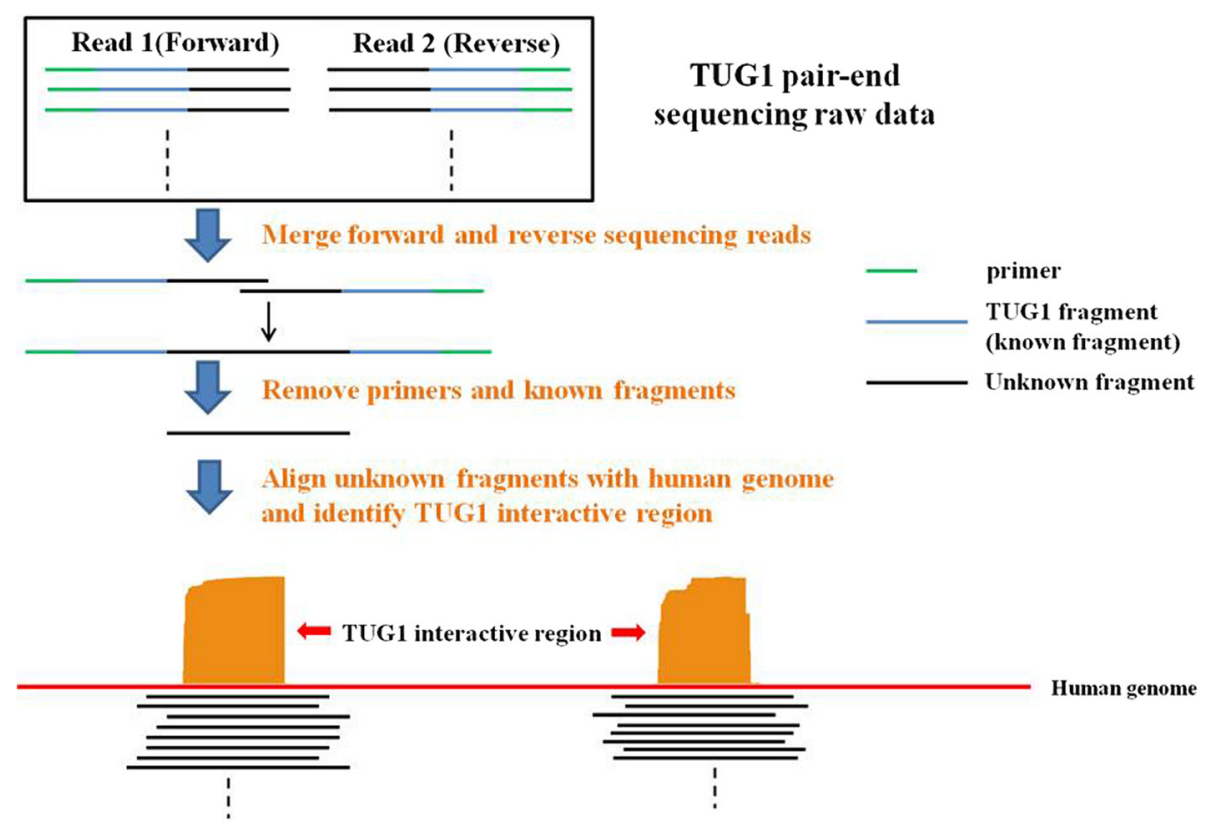

Fig. 3 Analysis flowchart for the TUG1 4C-sequencing data

Fig. 4a. Every candidate interaction region was examined to determine whether Hind III and CviQI digest sites were close to the 5' and 3' end of the region (Additional file 6: Table S5). Among the remaining candidate genes, the protein-coding gene CELF1 had one of the highest abundances. Because the other protein coding gene, MORC2, was located in the same chromosome as TUG1, the possibility of self-ligation cannot be excluded. Moreover, previous studies have reported that TUG1 regulation is involved in in-trans regulation. Therefore, an additional experiment was performed on CELF1 to confirm the interaction between TUG1 and CELF1. The interaction region of CELF1 located at chr11:47496189-47496438, and the read distribution of CELF1 are illustrated in Fig. 4b. The levels of CELF1 expression were detected using qRT-PCR in the TUG1 knockdown H520 cells. CELF1 was found to

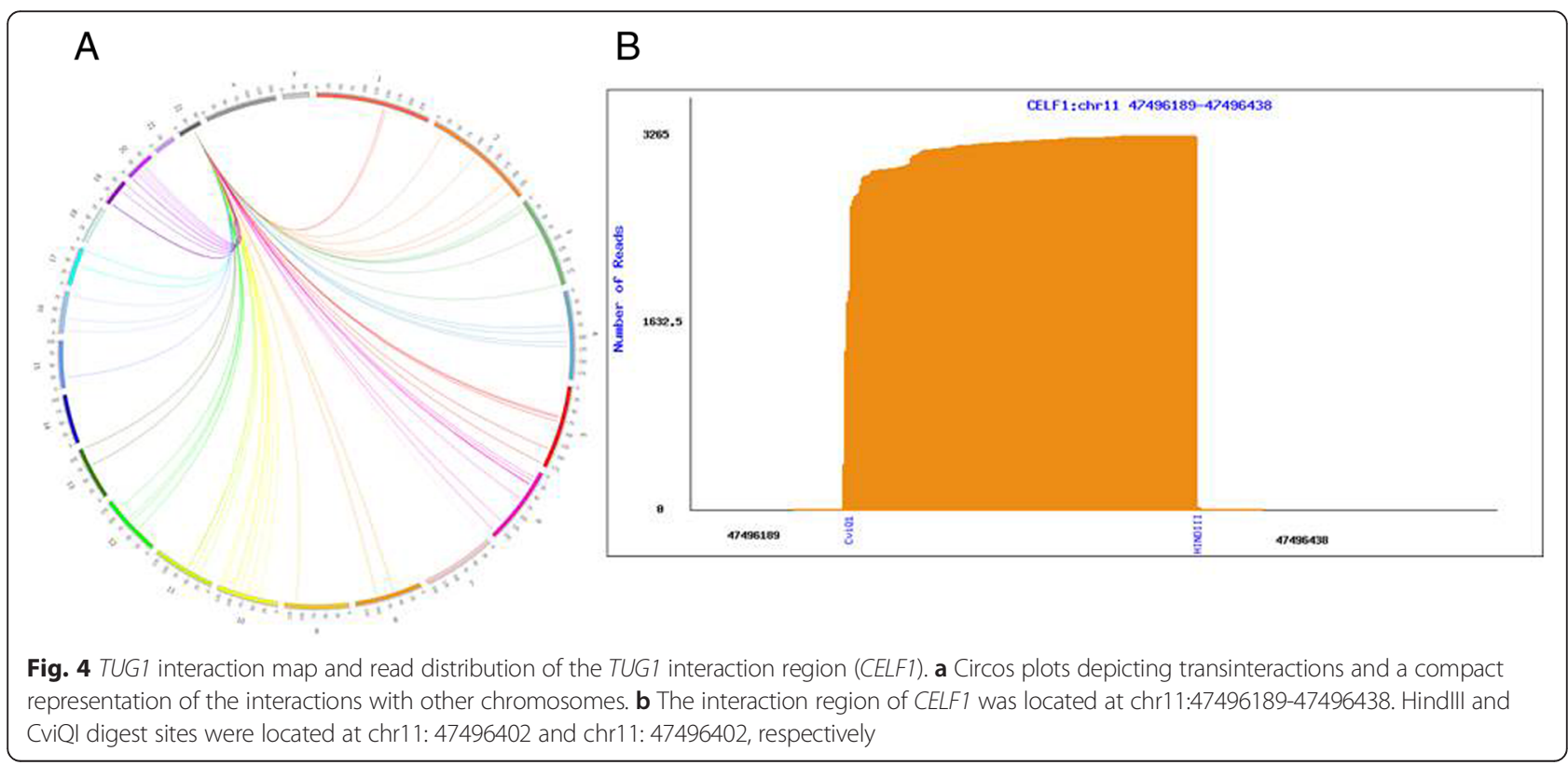


be significantly upregulated in these cells $(p<0.05$, Fig. 5a). Because lncRNAs might be involved in the gene regulatory network by guiding chromatin-modifying complexes to their sites of action, some lncRNAs may be central to the epigenetic control of gene expression [25]. TUG1 was previously shown to bind to PRC2 and epigenetically repress the expression of genes involved in cell-cycle regulation [26]. Yang et al. reported that TUG1 represses E2Fregulated growth-control genes (MCM3, PCNA, and $M S H 2$ ) by relocating them to polycomb bodies [16]. Zhang et al. screened the HOX family in TUG1 knockdown NSCLC cells, finding that TUG1 can negatively regulate HOX7 by binding to PRC2 [15]. Therefore, we investigated whether PRC2 is involved in the regulation of TUG1 on CELF1. We used the RIP assay to examine the association of PRC2 and TUG1 RNA. The binding of TUG1 RNA and enhancer of zeste protein 2/embryonic ectoderm development (EZH2/EED), which is crucial for PRC2, was validated in the RIP assay (Fig. 5b). Furthermore, we designed six sets of primers for the promotor region of CELF1 and found that EZH2/EED was bound to the sequences within $992 \mathrm{bp}$ upstream of the transcript start site (Fig. 5c). The spatial proximity of TUG1 and CELF1 chromatin segments was revealed through TUG1 $4 \mathrm{C}$-sequencing and bioinformatic analysis. The interaction between TUG1 RNA and CELF1 was confirmed by the negative regulation of TUG1 RNA on CELF1 and the occupancy of TUG1 RNA/PRC2 in the promoter region of CELF1.

\section{Discussion}

TUG1 was first identified as a noncoding RNA upregulated by taurine in developing retinal cells. Several genes related to cell-death pathways (AIF, Alix/AIP1, NIP3, and NAPOR) were shown to be upregulated in TUG1 knockdown cells and apoptosis was found to increase, suggesting that TUG1 is involved in cell survival [11]. Studies on human cancers have concluded that TUG1 is overexpressed in urothelial carcinoma of the bladder and is associated with high-grade and advanced-stage diseases. Inhibition of cell proliferation and induction of apoptosis were observed in TUG1 knockdown bladder urothelial carcinoma T24 and 5637 cells [12]. Similar results have been noted in osteosarcoma and esophageal squamous cell carcinomas [13, 14]. In lung cancer, TUG1 was shown to be downregulated in NSCLC, and TUG1 expression was shown to be significantly lower in advanced-stage diseases and larger tumors [15]. In our NSCLC cohort, TUG1 was downregulated in lung cancer tissues compared with the nontumor tissues. In addition, TUG1 levels were significanly lower in the patients who were male, smokers, or had poorly differentiated tumors. No significant difference in TUG1 levels was observed
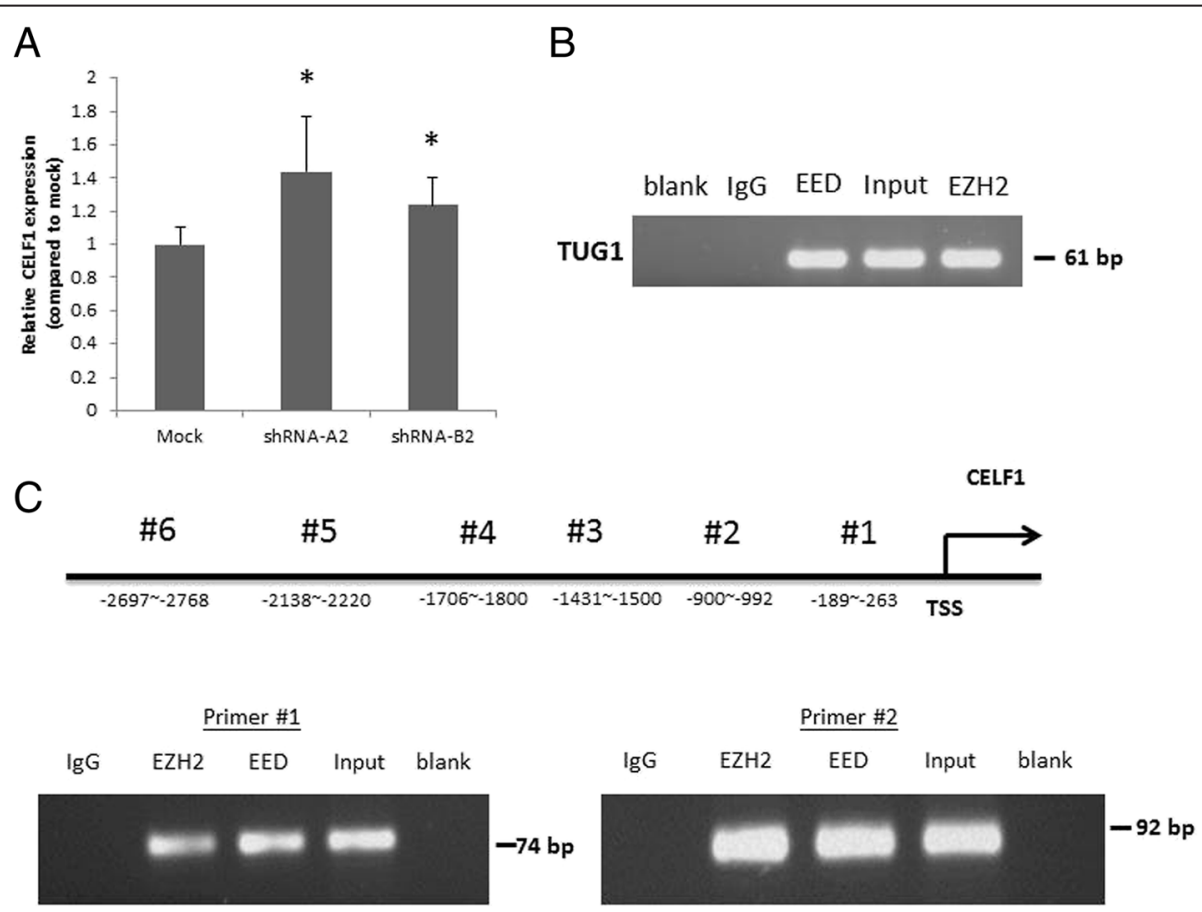

Fig. 5 Validation of interactions between CELF1 and TUG1. a Relative expressions of CELF1 in the mock, shRNA-A2, or shRNA-B2 transfected H520 cells were detected through qRT-PCR in three independent experiments. Significant CELF1 upregulation was noted in the TUG1 knockdown H520 cells. ( $\left.{ }^{*} p<0.05\right)$ (b) RIP experiments were performed on H520 cells by using EED, EZH2, and IgG. Coprecipitated RNA was tested through qRT-PCR for TUG1. c Six primer sets were designed for ChIP experiments on EZH2 and EED of the promotor regions of CELF1. qPCR was used to determine the quantitation of ChIP assays. Representative images of the three independent experiments are shown 
among patients with different disease stages; this could be attributed to the limited numbers of patients with advanced-stage disease in this study (four patients with stage IV disease).

LncRNAs have been described in the processes of gene silencing, imprinting, and gene activation [8]. In our study, CELF1 was identified through a $4 \mathrm{C}$-sequencing approach; the TUG1-involved regulation of CELF1 was confirmed through MTT assay, RIP, and DNA ChIP experiments. The $4 \mathrm{C}$ techniques, which are based on $3 \mathrm{C}$ techniques, enable identifying physical interactions between chromatin segments $[27,28]$. In addition to $3 \mathrm{C}$ procedures, $4 \mathrm{C}$ procedures also involve a second restrictive enzyme reaction; $4 \mathrm{C}$ procedures introduce a circularization step after the reversal of the cross-linking, increase the likelihood of promoting intermolecular ligation events, and generate high-resolution interaction maps [29]. Previous research revealed a strong connection between the spatial organization of chromatin and gene regulation, particularly the promotor-enhancer contacts induced by chromatin looping [30]. Kaufmann et al. conducted a large-scale investigation of the interchromosomal segment and gene contact networks, and showed that coexpression and functional similarity correlate with spatial proximity [31]. In the present study, we applied 4C procedures followed by nextgeneration sequencing (NGS) and bioinformatic analysis to investigate the possible interactions of TUG1 with other elements in the genome and found this approach effective for identifying IncRNA regulation targets.

CELF1 belongs to the CELF family (CUG-BP, Elav-like family). First recognized in human cells as a nuclear RNAbinding protein, CELF1 is expressed broadly in human tissues, such as the heart, skeletal muscles, and brain [32]. It is expressed in both the nucleus and cytoplasm, and is involved in pre-mRNA alternative splicing, RNA editing in the nucleus, and deadenylation, RNA decay, and translation in the cytoplasm [32]. CELF1 was shown to interact with the 5'-region of $c / e b p \beta m R N A$, and it is associated with polysomes that translate low molecular weight isoforms of C/EBP $\beta$ [33]. In chronic myeloid leukemia, $C E L F 1$ is repressed, resulting in a decrease of $C / E B P \beta$ isoforms, particularly LAP2. Imatinib (STI571) treatment can reinduce $C / E B P \beta$ expression that appears to depend on CELF1 expression and the integrity of the CUG-rich intercistronic region of $c / e b p \beta m R N A$ [34]. In oral squamous cancer cells, CELF1 is overexpressed, and CELF1 depletion reduces proliferation and increases apoptosis in these cells [35]. Wu et al. reported CELF1 overexpression in NSCLC and that siRNA-mediated silencing of CELF1 markedly reduced the survival rate and colony formation of lung cancer cells [36]. Although the roles of CELF1 in embryonic development and carcinogenesis were revealed, the upstream regulation of CELF1 has been overlooked. Our results reveal that $C E L F 1$ is negatively regulated by TUG1 in NSCLC cells. Microarray in TUG1 knockdown murine retinal cells showed upregulation of the other members of the same CELF subfamily, CELF2 (NAPOR) [11]. CELF1 and CELF2 exhibit conserved, partially overlapping, developmental-stage, tissue-specific expression [37]. In murine eyes, CELF1 protein expression is higher in the lens, whereas CELF2 protein is more highly expressed in the retinae [37]. This may explain why Young et al. found no linkage between TUG1 and CELF1-because that study focused on murine retinal cells. Therefore, CELF1 and CELF2 may share similar regulatory control mechanisms in a tissue-specific pattern through TUG1/PRC2 interaction.

\section{Conclusion}

LncRNA TUG1 is downregulated in NSCLC. We found that this downregulation is associated with sex, smoking status, and the differentiation grade of patients with NSCLC. Our study demonstrates a novel target of TUG1 and validates the interactions of TUG1/PRC2/CELF1 in NSCLC cells. The application of $4 \mathrm{C}$ techniques enabled a genome-wide search for lncRNA targets, the findings of which may facilitate future studies of lncRNAs. Our results may be used in the development of new treatment modalities targeting TUG1/PRC2/CELF1 interactions in patients with NSCLC.

\section{Additional files}

Additional file 1: RNA extraction, cDNA generation and quantitative PCR. (DOCX $19 \mathrm{~kb})$

Additional file 2: RIP and DNA ChIP. (DOCX $15 \mathrm{~kb}$ )

Additional file 3: Table S1. Sequences of primers for ChIP assay of the promoter region in CELF1. (DOCX $19 \mathrm{~kb}$ )

Additional file 4: Table S2. Clinical parameters and relative expressions of TUG1 in NSCLC. (DOCX $17 \mathrm{~kb}$ )

Additional file 5: Figure S1. Digestion and PCR amplification of the TUG1-4C procedure. (A) Agarose gel (0.8\%, wt/vol) of the undigested (left lane) and primary digested (right lane) sample (HindIII, six-cutter) (B) Agarose gel $(2.0 \%$, wt/vol) of the secondary digested sample (CViQl four-cutter). (C) Inverse PCR amplification of the 4C samples generated amplified sequences of a wide range of sizes. (JPG $26 \mathrm{~kb}$ )

Additional file 6: Table S3. Candidate genes of TUG1 4C-sequencing. Table S4. Gene fragments of TUG1 4C-sequencing. Table S5. Hind III and $\mathrm{CViQ}$ ligest sites were proximal to the $5^{\prime}$ and $3^{\prime}$ end of the region. (XLSX $28 \mathrm{~kb}$ )

\footnotetext{
Abbreviations

3C, chromosome conformation capture; 4C, circular chromosome conformation capture; ALK, anaplastic lymphoma kinase; CDNA, complementary DNA; CELF1, CUGBP and Elav-like family member 1; ECOG PS, Eastern Cooperative Oncology Group performance status; EED, embryonic ectoderm development; EGFR, epidermal growth factor receptor; EZH2, zeste protein 2; GAPDH, glyceraldehyde-3-phosphate dehydrogenase; IncRNAs, long noncoding RNAs; MTT, 3-(4,5-dimethylthiazol-2-yl)-2,5-diphenyltetrazolium bromide; NGS, next-generation sequencing; NSCLC, non-small cell lung cancer; $P C R$, polymerase chain reaction; $q R T-P C R$, quantitative reverse transcription polymerase chain reaction; RTK, receptor tyrosine kinase; SCLC, small-cell lung cancer; TUG1, taurine-upregulated gene 1
} 


\section{Acknowledgements}

This study was supported by grants from the Ministry of Science and Technology (NSC 102-2314-B-037-059- and NSC 99-2320-B-039-038-MY3), Kaohsiung Medical University Hospital (KMUH100-0 M21 and KMUH1022R30), and China Medical University (CMU103-BC-7). The authors thank the Statistical Analysis Laboratory, Department of Medical Research, Kaohsiung Medical University Hospital, and Kaohsiung Medical University for their help. The authors thank Dr. Wei-Chi Wang (Health GeneTech Corporation, Taoyuan, Taiwan) for bioinformatic analysis of 4C-sequencing data.

\section{Funding}

This study was supported by grants from the Ministry of Science and Technology (NSC 102-2314-B-037-059- and NSC 99-2320-B-039-038-MY3), Kaohsiung Medical University Hospital (KMUH100-0 M21 and KMUH102-2R30), and China Medical University (CMU103-BC-7).

\section{Availability of data and material}

The datasets supporting the conclusions of this article are included within the article and its additional files.

\section{Authors' contributions}

JGC and TCL conceived of the study and participated in its design and coordination. HDH participated in the design of the study and conducted the bioinformatic analysis. Clinical data acquisition and analysis were carried out by CCC and PCL. YSC, JCY and CCL carried out the laboratory experiments. PCL wrote this manuscript. WHC helped to draft the manuscript. All authors have read and approve the final version of the manuscript.

\section{Authors' information}

Not applicable.

\section{Competing interests}

The authors declare that they have no competing interests.

\section{Consent for publication}

Not applicable.

\section{Ethics approval and consent to participate}

This research was approved by the Institutional Review Board of Kaohsiung Medical University Hospital (KMUH-IRB-980524), and informed consent was obtained from all participants.

\section{Author details \\ 'Graduate Institute of Clinical Medicine, Kaohsiung Medical University, No. 100, Shih-Chuan 1st Road, Kaohsiung, Taiwan. ${ }^{2}$ Epigenome Research Center, China Medical University Hospital, No. 2, Yuh-Der Road, Taichung, Taiwan. ${ }^{3}$ Department of Biological Science and Technology, Institute of Bioinformatics and Systems Biology, National Chiao Tung University, Hsinchu, Taiwan. ${ }^{4}$ Department of Laboratory Medicine, China Medical University Hospital, Taichung, Taiwan. ${ }^{5}$ School of Medicine, China Medical University, Taichung, Taiwan. ${ }^{6}$ Division of Hematology and Oncology, Department of Internal Medicine, Kaohsiung Medical University Hospital, Kaohsiung Medical University, Kaohsiung, Taiwan. ${ }^{7}$ Department of Laboratory Medicine, Kaohsiung Medical University Hospital, Kaohsiung Medical University, Kaohsiung, Taiwan. ${ }^{8}$ Department of Pediatrics, School of Medicine, College of Medicine, Kaohsiung Medical University, Kaohsiung, Taiwan. ${ }^{9}$ Division of Hematology and Oncology, Department of Pediatrics, Kaohsiung Medical University Hospital, Kaohsiung, Taiwan. ${ }^{10}$ Division of Chest Medicine, Department of Internal Medicine, Changhua Christian Hospital, Changhua, Taiwan.}

\section{Received: 29 October 2015 Accepted: 15 July 2016} Published online: 02 August 2016

\section{References}

1. McErlean A, Ginsberg MS. Epidemiology of lung cancer. Semin Roentgenol. 2011;46(3):173-7.

2. Reck M, Heigener DF, Mok T, Soria JC, Rabe KF. Management of non-smallcell lung cancer: recent developments. Lancet. 2013;382(9893):709-19.
3. Haghgoo SM, Allameh A, Mortaz E, Garssen J, Folkerts G, Barnes PJ, et al. Pharmacogenomics and targeted therapy of Cancer: focusing on non-small cell lung Cancer. Eur J Pharmacol. 2015;754:82-91.

4. Yang L, Froberg JE, Lee JT. Long noncoding RNAs: fresh perspectives into the RNA world. Trends Biochem Sci. 2014;39(1):35-43.

5. Guttman M, Donaghey J, Carey BW, Garber M, Grenier JK, Munson G, et al lincRNAs act in the circuitry controlling pluripotency and differentiation. Nature. 2011;477(7364):295-300.

6. Sheik Mohamed J, Gaughwin PM, Lim B, Robson P, Lipovich L. Conserved long noncoding RNAs transcriptionally regulated by Oct4 and Nanog modulate pluripotency in mouse embryonic stem cells. RNA. 2010;16(2):324-37.

7. Tsai MC, Spitale RC, Chang HY. Long intergenic noncoding RNAs: new links in cancer progression. Cancer Res. 2011;71(1):3-7.

8. Wapinski O, Chang HY. Long noncoding RNAs and human disease. Trends Cell Biol. 2011;21(6):354-61.

9. Bhan A, Mandal SS. Long noncoding RNAs: emerging stars in gene regulation, epigenetics and human disease. Chem Med Chem. 2014;9(9):1932-56.

10. $\mathrm{Li} \mathrm{CH}$, Chen Y. Targeting long non-coding RNAs in cancers: progress and prospects. Int J Biochem Cell Biol. 2013;45(8):1895-910.

11. Young $T L$, Matsuda $T$, Cepko CL. The noncoding RNA taurine upregulated gene 1 is required for differentiation of the murine retina. Curr Biol. 2005;15(6):501-12

12. Han Y, Liu Y, Gui Y, Cai Z. Long intergenic non-coding RNA TUG1 is overexpressed in urothelial carcinoma of the bladder. J Surgical Oncol. 2013;107(5):555-9.

13. Zhang $Q$, Geng $P L$, Yin $P$, Wang $X L$, Jia JP, Yao J. Down-regulation of long non-coding RNA TUG1 inhibits osteosarcoma cell proliferation and promotes apoptosis. Asian Pac J Cancer Prev. 2013;14(4):2311-5.

14. Xu Y, Wang J, Qiu M, Xu L, Li M, Jiang F, et al. Upregulation of the long noncoding RNA TUG1 promotes proliferation and migration of esophageal squamous cell carcinoma. Tumour Biol. 2015:36(3):1643-51.

15. Zhang EB, Yin DD, Sun M, Kong R, Liu XH, You LH, et al. P53-regulated long non-coding RNA TUG1 affects cell proliferation in human non-small cell lung cancer, partly through epigenetically regulating HOXB7 expression. Cell Death Dis. 2014;5:e1243.

16. Yang L, Lin C, Liu W, Zhang J, Ohgi KA, Grinstein JD, et al. ncRNA- and PC2 methylation-dependent gene relocation between nuclear structures mediates gene activation programs. Cell. 2011;147(4):773-88.

17. Ponting CP, Oliver PL, Reik W. Evolution and functions of long noncoding RNAs. Cell. 2009;136(4):629-41.

18. Stadhouders R, Kolovos P, Brouwer R, Zuin J, van den Heuvel A, Kockx C, et al. Multiplexed chromosome conformation capture sequencing for rapid genome-scale high-resolution detection of long-range chromatin interactions. Nat Protoc. 2013;8(3):509-24.

19. Zhao Z, Tavoosidana G, Sjolinder M, Gondor A, Mariano P, Wang S, et al. Circular chromosome conformation capture (4C) uncovers extensive networks of epigenetically regulated intra- and interchromosomal interactions. Nature Gen. 2006;38(11):1341-7.

20. Altschul SF, Gish W, Miller W, Myers EW, Lipman DJ. Basic local alignment search tool. J Mol Biol. 1990;215(3):403-10.

21. Langmead B, Trapnell C, Pop M, Salzberg SL. Ultrafast and memory-efficient alignment of short DNA sequences to the human genome. Genome Biol. 2009;10(3):R25.

22. Williams Jr RL, Starmer J, Mugford JW, Calabrese JM, Mieczkowski P, Yee D, et al. fourSig: a method for determining chromosomal interactions in $4 \mathrm{C}$ Seq data. Nucleic Acids Res. 2014;42(8):e68.

23. Orom UA, Derrien T, Beringer M, Gumireddy K, Gardini A, Bussotti G, et al. Long noncoding RNAs with enhancer-like function in human cells. Cell. 2010;143(1):46-58.

24. Wang KC, Yang YW, Liu B, Sanyal A, Corces-Zimmerman R, Chen Y, et al. A long noncoding RNA maintains active chromatin to coordinate homeotic gene expression. Nature. 2011;472(7341):120-4.

25. Mattick JS, Amaral PP, Dinger ME, Mercer TR, Mehler MF. RNA regulation of epigenetic processes. Bioessays. 2009;31(1):51-9.

26. Khalil AM, Guttman M, Huarte M, Garber M, Raj A, Rivea Morales D, et al. Many human large intergenic noncoding RNAs associate with chromatinmodifying complexes and affect gene expression. Proc Natl Acad Sci U S A. 2009;106(28):11667-72 
27. Dekker J, Rippe K, Dekker M, Kleckner N. Capturing chromosome conformation. Science. 2002;295(5558):1306-11.

28. Splinter E, Grosveld F, de Laat W. 3 C technology: analyzing the spatial organization of genomic loci in vivo. Methods Enzymol. 2004;375:493-507.

29. Gondor A, Rougier C, Ohlsson R. High-resolution circular chromosome conformation capture assay. Nat Protoc. 2008;3(2):303-13.

30. Jin F, Li Y, Dixon JR, Selvaraj S, Ye Z, Lee AY, et al. A high-resolution map of the three-dimensional chromatin interactome in human cells. Nature. 2013; 503(7475):290-4

31. Kaufmann S, Fuchs C, Gonik M, Khrameeva EE, Mironov AA, Frishman D. Inter-chromosomal contact networks provide insights into Mammalian chromatin organization. PLoS One. 2015;10(5):e0126125.

32. Dasgupta T, Ladd AN. The importance of CELF control: molecular and biological roles of the CUG-BP, Elav-like family of RNA-binding proteins. Wiley Interdiscip Rev RNA. 2012;3(1):104-21.

33. Timchenko NA, Welm AL, Lu X, Timchenko LT. CUG repeat binding protein (CUGBP1) interacts with the $5^{\prime}$ region of C/EBPbeta mRNA and regulates translation of C/EBPbeta isoforms. Nucleic Acids Res. 1999;27(22):4517-25.

34. Guerzoni C, Bardini M, Mariani SA, Ferrari-Amorotti G, Neviani P, Panno ML, et al. Inducible activation of $C E B P B$, a gene negatively regulated by $B C R /$ $A B L$, inhibits proliferation and promotes differentiation of $B C R / A B L-$ expressing cells. Blood. 2006;107(10):4080-9.

35. Talwar S, Balasubramanian S, Sundaramurthy S, House R, Wilusz CJ, Kuppuswamy D, et al. Overexpression of RNA-binding protein CELF1 prevents apoptosis and destabilizes pro-apoptotic mRNAs in oral cancer cells. RNA Biol. 2013:10(2):277-86.

36. Wu LN, Xue YJ, Zhang LJ, Ma XM, Chen JF. Si-RNA mediated knockdown of CELF1 gene suppressed the proliferation of human lung cancer cells. Cancer Cell Int. 2013;13(1):115.

37. Blech-Hermoni Y, Stillwagon SJ, Ladd AN. Diversity and conservation of CELF1 and CELF2 RNA and protein expression patterns during embryonic development. Dev Dyn. 2013;242(6):767-77.

\section{Submit your next manuscript to BioMed Central and we will help you at every step:}

- We accept pre-submission inquiries

- Our selector tool helps you to find the most relevant journal

- We provide round the clock customer support

- Convenient online submission

- Thorough peer review

- Inclusion in PubMed and all major indexing services

- Maximum visibility for your research

Submit your manuscript at www.biomedcentral.com/submit 